Comment. Math. Helv. 79 (2004) 317-340

0010-2571/04/020317-24

DOI $10.1007 / \mathrm{s} 00014-004-0803-3$
(C) 2004 Birkhäuser Verlag, Basel

Commentarii Mathematici Helvetici

\title{
Families of strong KT structures in six dimensions
}

\author{
Anna Fino, Maurizio Parton and Simon Salamon
}

\begin{abstract}
This paper classifies Hermitian structures on 6-dimensional nilmanifolds $M=\Gamma \backslash G$ for which the fundamental 2 -form is $\partial \bar{\partial}$-closed, a condition that is shown to depend only on the underlying complex structure $J$ of $M$. The space of such $J$ is described when $G$ is the complex Heisenberg group, and explicit solutions are obtained from a limaçon-shaped curve in the complex plane. Related theory is used to provide examples of various types of Ricci-flat structures.
\end{abstract}

Mathematics Subject Classification (2000). 53C55; 32G05, 17B30, 81T30.

Keywords. Hermitian metric, complex structure, connection, torsion, nilmanifold.

\section{Introduction}

Let $(M, J, g)$ be a Hermitian manifold. There is a 1-parameter family of canonical Hermitian connections on $M$ which can be distinguished by properties of the torsion tensor $T[13,30]$. In particular, there is a unique connection $\nabla$ satisfying $\nabla g=0, \nabla J=0$ for which $g(X, T(Y, Z))$ is totally skew-symmetric. The resulting 3 -form can then be identified with $J d \Omega$, where $\Omega$ is the fundamental 2-form defined by (1). This connection was used by Bismut [4] to prove a local index formula for the Dolbeault operator when the manifold is non-Kähler, and is the subject of a number of other interesting results $[16,20]$. The properties of such a connection give rise to what is loosely called 'Kähler with torsion geometry', and if $J d \Omega$ is closed but non-zero then $g$ is called a strong KT metric. Such metrics have applications in type II string theory and in 2-dimensional supersymmetric $\sigma$-models $[11,19,29]$.

In four real dimensions, a metric satisfying the strong KT condition is 'standard' in the terminology of Gauduchon [12]. One can be found in the conformal class of any given Hermitian metric on a compact manifold. But the theory is very different in higher dimensions. Even-dimensional compact Lie groups provide a natural class of strong $\mathrm{KT}$ structures [28]. In this case, one may choose $J$ to be a left-invariant complex structure and $g$ to be a compatible bi-invariant metric. Then $\nabla$ is the flat connection with skew-symmetric torsion $g(X,[Y, Z])$ corresponding to an invariant 3 -form on the Lie algebra. It is therefore natural to 
investigate the situation with regard to other groups.

In this paper, we study KT geometry on 6-dimensional nilmanifolds in which $J$ and $g$ arise from corresponding left-invariant tensors. If $G$ is a simply-connected nilpotent Lie group, and if the structure equations of its Lie algebra are rational, then there exists a discrete subgroup $\Gamma$ of $G$ for which $M=\Gamma \backslash G$ is compact [22, 25]. Any left-invariant complex structure on $G$ will pass to a complex structure $J$ on $M$ but, unless $G$ is abelian, the $\partial \bar{\partial}$-lemma is not valid for $J$ and in particular there is no compatible Kähler metric [3, $7,17,24]$. As we explain in $\S 6$, there may or may not be invariant pseudo-Kähler metrics on $(M, J)$.

Eighteen of the thirty-four classes of real 6-dimensional nilpotent Lie algebras $\mathfrak{g}$ admit a complex structure. Exactly four of these classes, all of them 2-step with $b_{1} \geqslant 4$ and including the case in which $\mathfrak{g}$ underlies the complex Heisenberg algebra, give rise to strong KT metrics. Given that compact nilmanifolds with a strong KT structure exist, it is perhaps surprising that there are so few classes. The classification over $\mathbb{R}$ is accomplished in $\S 3$, after an analysis of the relevant structure equations over $\mathbb{C}$ in $\S \S 1,2$. A matrix formalism for describing $(1,1)$-forms is introduced in an attempt to make the calculations of this paper rather more enlightening. A striking feature of our classification is that the existence of a strong KT structure depends only on the complex structure of $\mathfrak{g}$, and this poses the question of understanding the solutions as a subset of an appropriate moduli space of complex structures.

With this aim, we proceed to a detailed study of the strong KT equations when $G$ is the complex Heisenberg group and $M=\Gamma \backslash G$ is the Iwasawa manifold. It is easy to check that none of the standard complex structures [1] on $G$ are strong KT, so we were intrigued to discover which ones are. According to the third author's joint paper with Ketsetzis [21], essential features of an invariant complex structure $J$ on $M$ depend on $\mathbf{X} \overline{\mathbf{X}}$, where $\mathbf{X}$ is a $2 \times 2$ matrix representing the induced action of $J$ on $M / T^{2} \cong T^{4}$. In $\S 4$, we prove that the strong $\mathrm{KT}$ condition constrains the eigenvalues of $\mathbf{X} \overline{\mathbf{X}}$ to be complex conjugates lying on a curve in the complex plane. We interpret this result in terms of the action of the automorphism group of $\mathfrak{g}$ in $\S 5$, and this leads to an explicit description of the solution space. An analogous study can probably be carried out when $G=H_{3} \times H_{3}$ is the product of real Heisenberg groups, using methods from [14].

A Hermitian manifold is called conformally balanced if the Lee 1-form $\theta$ (the 'trace' of $d \Omega$ ) is exact. The study of such structures in relation to the Bismut connection $\nabla$ is motivated by work of [26], though there are less subtleties in our invariant context. It was observed in [2] that the vanishing of $\theta$ is complementary to the SKT condition (see $\S 1$ ), and we begin the final section by discussing some known facts concerning the situation in which the holonomy of $\nabla$ reduces to $S U(n)$. We list some 6-dimensional Lie algebras giving rise to nilmanifolds admitting such a reduction, and give an example of a pseudo-Riemannian metric with zero Ricci tensor. 
Acknowledgment. Thanks are due to H. Pedersen and A.F. Swann for help and encouragement, and to S. Ivanov for suggesting Proposition 6.1. This paper was conceived whilst the first two authors were visiting Odense, and all three are members of EDGE, Research Training Network HPRN-CT-2000-00101, supported by the European Human Potential Programme.

\section{Complex structure equations}

Let $(M, J, g)$ be a Hermitian manifold of real dimension $2 n$. We shall regard the complex structure $J$ as the primary object, so the Riemannian metric $g$ is chosen to render $J$ orthogonal. The fundamental 2 -form $\Omega$ is then defined by

$$
g(X, Y)=\Omega(X, J Y)
$$

and has type $(1,1)$ relative to $J$. The Hermitian structure is Kähler if and only if $d \Omega=0$, which is equivalent to the vanishing of $\partial \Omega=(d \Omega)^{2,1}$.

Somewhat unconventionally, we set

$$
\square=\frac{1}{2} i d J d .
$$

This operator acts as $\partial \bar{\partial}$ on forms of type $(p, p)$, which it maps to forms of type $(p+1, p+1)$. We shall only be concerned with the case $p=1$.

Definition 1.1. We shall say that the Hermitian manifold $(M, J, g)$ is 'strong KT' or more briefly 'SKT' if $\square \Omega=0$ but $d \Omega \neq 0$.

Observe that our definition of SKT excludes the Kähler case.

We wish to combine the notion of SKT with that of an invariant Hermitian structure on a nilmanifold. First recall the definition of nilpotency for a Lie algebra $\mathfrak{g}$. The descending central series of $\mathfrak{g}$ is the chain of ideals defined inductively by $\mathfrak{g}^{0}=\mathfrak{g}$ and $\mathfrak{g}^{i}=\left[\mathfrak{g}^{i-1}, \mathfrak{g}\right]$ for $i \geqslant 1$. Then $\mathfrak{g}$ is nilpotent if $\mathfrak{g}^{s}=0$ for some $s$. If, in addition, $\mathfrak{g}^{s-1} \neq 0$ then $\mathfrak{g}$ is said to be $s$-step.

Let $\mathfrak{g}$ be a real $2 n$-dimensional nilpotent Lie algebra. Assigning an almost complex structure $J: \mathfrak{g} \rightarrow \mathfrak{g}$ is equivalent to choosing an $n$-dimensional subspace $\Lambda$ of $\mathfrak{g}_{c}^{*}$ such that $\Lambda \cap \bar{\Lambda}=\{0\}$. For the purpose of this paper, we shall call such a subspace of $\mathfrak{g}_{c}^{*}$ 'maximally complex'. The endomorphism $J$ extends uniquely to a left-invariant almost complex structure (also denoted by $J$ ) on any Lie group $G$ with Lie algebra $\mathfrak{g}$. The subspace $\Lambda$ generates the space of $(1,0)$-forms relative to $J$, and this is a complex structure if and only if $\mathcal{I}(\Lambda)$ is a differential ideal. (We use $\mathcal{I}(S)$ to denote the ideal generated by a subset $S$ of the exterior algebra $\Lambda^{*} \mathfrak{g}$.)

By a 'nilmanifold with an invariant complex structure' we mean an evendimensional nilmanifold $\Gamma \backslash G$ endowed with a complex structure $J$ arising from g. It is important to note that $(G, J)$ will not in general be a complex Lie group. 
Theorem 1.2. Let $M=\Gamma \backslash G$ be a 6-dimensional nilmanifold with an invariant complex structure $J$. Then the SKT condition is satisfied by either all invariant Hermitian metrics $g$ or by none. Indeed, it is satisfied if and only if $J$ has a basis $\left(\alpha^{i}\right)$ of invariant $(1,0)$-forms such that

$$
\left\{\begin{array}{l}
d \alpha^{1}=0 \\
d \alpha^{2}=0 \\
d \alpha^{3}=A \alpha^{\overline{1} 2}+B \alpha^{\overline{2} 2}+C \alpha^{1 \overline{1}}+D \alpha^{1 \overline{2}}+E \alpha^{12}
\end{array}\right.
$$

where $A, B, C, D, E$ are complex numbers such that

$$
|A|^{2}+|D|^{2}+|E|^{2}+2 \operatorname{Re}(\bar{B} C)=0 .
$$

We indicate $\alpha^{i} \wedge \alpha^{\bar{j}}$ by $\alpha^{i \bar{j}}$ (or $-\alpha^{\bar{j} i}$ ), and use similar notation for forms of arbitrary degree. Thus, the symbol $\alpha$ stands more for the choice of basis than for an individual element.

The third equation in (2) means that

$$
d \alpha^{3} \in \bigwedge^{2}\left\langle\alpha^{1}, \alpha^{\overline{1}}, \alpha^{2}, \alpha^{\overline{2}}\right\rangle .
$$

and the resulting complex structure $J$ is of 'nilpotent' type in the language of [6]. Observe that the system (2) automatically satisfies $d^{2}=0$ and therefore defines a Lie algebra irrespective of the values of $A, B, C, D, E$. The resulting isomorphism classes are listed in $\S 3$, where we distinguish those compatible with (3).

We shall divide the proof of Theorem 1.2 into two parts. The second part is devoted to a mainly computational derivation of the structure equations (2), and is relegated to the next section. However, it is instructive to begin by assuming (2) and deducing (3) from it. This we do immediately, and in passing we shall see that the choice of metric is irrelevant.

Let $\Omega$ be the fundamental 2 -form of some $J$-Hermitian metric, and set

$$
\Omega=\sum_{i, j=1}^{3} x_{i \bar{j}} \alpha^{i \bar{j}}
$$

where $x_{i \bar{j}} \in \mathbb{C}$ are constant coefficients with $\overline{x_{j \bar{i}}}=-x_{i \bar{j}}$. The positive definiteness of $g$ implies that the restriction of $\Omega$ to any complex line is non-zero. Equivalently,

$$
\Omega(V, J \bar{V})=g(V, \bar{V})>0
$$

for any vector $V \neq 0$ in the complexified tangent space.

Given that $J d \alpha^{3}$ differs from $d \alpha^{3}$ by changing the sign of $E$, an easy calculation gives

$$
J d \alpha^{3} \wedge d \alpha^{\overline{3}}=\left(|A|^{2}+B \bar{C}+C \bar{B}+|D|^{2}+|E|^{2}\right) \alpha^{1 \overline{1} 2 \overline{2}} .
$$

The vanishing of this 4 -form is precisely (3), which can now be deduced from (2) via

Lemma 1.3. Given (2), $(M, J, g)$ is SKT for any invariant Hermitian metric $g$ if and only if $J d \alpha^{3} \wedge d \alpha^{\overline{3}}=0$. 
Proof. Observe that $x_{3 \overline{3}} \neq 0$. Indeed, if $\left(V_{i}\right)$ is a basis of $(1,0)$-vectors dual to $\left(\alpha^{i}\right)$ then (5) implies that $-i x_{3 \overline{3}}=\Omega\left(V_{3}, J \bar{V}_{3}\right)>0$. On the other hand, all the terms in (4), with the exception of $x_{3 \overline{3}} \alpha^{3 \overline{3}}$, are eliminated by two differentiations. Thus, the SKT condition is satisfied for any compatible metric if and only if $\square \alpha^{3 \overline{3}}=0$.

Let $\Psi=J d \alpha^{3} \wedge d \alpha^{\overline{3}}$, and observe that

$$
\begin{aligned}
2 \square \alpha^{3 \overline{3}}=d J d\left(i \alpha^{3 \overline{3}}\right) & =i d J\left(d \alpha^{3} \wedge \alpha^{\overline{3}}-\alpha^{3} \wedge d \alpha^{\overline{3}}\right) \\
& =d\left(J d \alpha^{3} \wedge \alpha^{\overline{3}}+\alpha^{3} \wedge J d \alpha^{\overline{3}}\right) \\
& =J d \alpha^{3} \wedge d \alpha^{3}+d \alpha^{3} \wedge J d \alpha^{3} \\
& =\Psi+J \Psi
\end{aligned}
$$

since $J^{2}=1$ on 2 -forms. But $\Psi$ is a form of type $(2,2)$ relative to $J$ (or any other almost complex structure on the real 4-dimensional space underlying $\left\langle\alpha^{1}, \alpha^{2}\right\rangle$ ), so $J \Psi=\Psi$.

The Lee form of a Hermitian manifold $(M, J, g)$ of real dimension $2 n$ is the 1 -form

$$
\theta=J * d * \Omega=-J d^{*} \Omega
$$

where $d^{*}$ is the formal adjoint of $d$ with respect to $g$. The formula $* \Omega=$ $\Omega^{n-1} /(n-1)$ ! implies that $d\left(\Omega^{n-1}\right)=\theta \wedge \Omega^{n-1}$. Equivalently,

$$
\varpi=d \Omega-\frac{1}{n-1} \theta \wedge \Omega
$$

satisfies $\varpi \wedge \Omega^{n-2}=0$, and is therefore a primitive form.

Almost Hermitian manifolds with $\theta=0$ have in the past been called semiKähler or cosymplectic, though a Hermitian structure is also called balanced if $\theta=0$. In this case, we are therefore talking about Hermitian manifolds of GrayHervella class $\mathcal{W}_{3}$ [15]. Since $\left\langle d^{*} \Omega, \sigma\right\rangle=\langle\Omega, d \sigma\rangle$ for all 1-forms $\sigma$, the vanishing of $\theta$ is equivalent to $\Omega$ being orthogonal to the image of $d$ in $\Lambda^{2} \mathfrak{g}^{*}$, a fact exploited in the study [1].

Under a conformal change $\tilde{g}=e^{2 f} g$, the Lee form transforms as

$$
\tilde{\theta}=\theta+2(n-1) d f .
$$

The Hermitian structure is conformally balanced if $\theta$ is exact, for in that case $f$ can be chosen so that $\tilde{\theta}=0$.

In real dimension 4 , the SKT condition is equivalent to $d^{*} \theta=0$. The following result is given in [2, Remark 1], but merits a proof in our setting.

Proposition 1.4. A Hermitian manifold $(M, J, g)$ of real dimension $2 n \geqslant 6$ can only be $S K T$ if $\theta \neq 0$.

Proof. Suppose that $\theta=0$. Since $d^{*} \Omega=-* d * \Omega$ and $* \Omega$ is proportional to $\Omega^{n-1}$, the $(2 n-1)$ form $\Phi=\Omega^{n-2} \wedge \partial \Omega$ vanishes. Thus $\partial \Omega$ is a primitive $(2,1)$-form, and

$$
0=\bar{\partial} \Phi=(n-2) \Omega^{n-3} \wedge \bar{\partial} \Omega \wedge \partial \Omega+\Omega^{n-2} \wedge \bar{\partial} \partial \Omega .
$$


Primitivity implies that $\Omega^{n-3} \wedge \overline{\partial \Omega} \wedge \partial \Omega$ is proportional to $\|\partial \Omega\|^{2}$, so $\bar{\partial} \partial \Omega=0$ now implies that $\partial \Omega=0$ and $M$ is Kähler. But this is excluded in Definition 1.1.

It is amusing to view this result in the light of Theorem 1.2. Suppose that $n=3$ and that (2) holds. Referring to (4), we may set

$$
x_{1 \overline{2}}=z, \quad x_{2 \overline{1}}=-\bar{z}, \quad x_{1 \overline{1}}=i x, \quad x_{2 \overline{2}}=i y
$$

with $x, y>0$ and $x y>|z|^{2}$ to reflect positivity. The condition that $\Omega$ be orthogonal to $d \alpha^{3}$ becomes

$$
A z+i(B x-C y)+D \bar{z}=0 .
$$

Proposition 1.4 implies that this is incompatible with the inequality

$$
|A|^{2}+|D|^{2}+2 \operatorname{Re}(\bar{B} C) \leqslant 0
$$

from (3) unless all the coefficients vanish. form

The incompatibility between (9) and (10) is clear if $\Omega$ assumes the standard

$$
\Omega_{0}=\frac{1}{2} i\left(\omega^{1 \overline{1}}+\omega^{2 \overline{2}}+\omega^{3 \overline{3}}\right) .
$$

For then $z=0, x=y$; thus $B=C$ and $\operatorname{Re}(B \bar{C})=|B|^{2}$. The general case is far less obvious, but follows by setting $B=y=1$ (which is no real restriction) and applying

Remark 1.5. Let $A, C, D, z \in \mathbb{C}$ be such that $x=C+i(A z+D \bar{z})$ is real. Then

$$
|A|^{2}+|D|^{2}+2 \operatorname{Re} C \leqslant 0 \quad \Rightarrow \quad x \leqslant|z|^{2} .
$$

To verify this, set $F=i(A z+D \bar{z})$ so that

$$
x=\operatorname{Re} C+\operatorname{Re} F \leqslant \operatorname{Re} C+|F| \leqslant-\frac{1}{2}\left(|A|^{2}+|D|^{2}\right)+(|A|+|D|)|z| .
$$

If $x>|z|^{2}$ then

$$
2|z|^{2}-2(|A|+|D|)|z|+|A|^{2}+|D|^{2}<0
$$

which (as a quadratic in $|z|$ with non-positive discriminant) is impossible.

\section{Reducing the coefficients}

This section is devoted to completing the proof of Theorem 1.2 by arriving at (2). Our starting point is [27, Theorem 1.3], which we mildly re-state as

Theorem 2.1. A maximally complex subspace $\Lambda$ of $\mathfrak{g}_{c}^{*}$ is the (1,0)-space of a complex structure if and only if $\Lambda$ has a basis $\left(\alpha^{i}\right)$ for which $d \alpha^{1}=0$ and $d \alpha^{i} \in$ $\mathcal{I}\left(\left\{\alpha^{1}, \ldots, \alpha^{i-1}\right\}\right)$ for $i \geqslant 2$. 
Recall that $\mathcal{I}$ stands for 'ideal'. In dimension 6 this result provides the generic structure equations

$$
\begin{aligned}
& d \alpha^{1}=0 \\
& d \alpha^{2}=a_{1} \alpha^{12}+a_{2} \alpha^{13}+a_{3} \alpha^{1 \overline{1}}+a_{4} \alpha^{1 \overline{2}}+a_{5} \alpha^{1 \overline{3}} \\
& d \alpha^{3}=b_{1} \alpha^{12}+b_{2} \alpha^{13}+b_{3} \alpha^{1 \overline{1}}+b_{4} \alpha^{1 \overline{2}}+b_{5} \alpha^{1 \overline{3}}+c_{1} \alpha^{23}+c_{2} \alpha^{2 \overline{1}}+c_{3} \alpha^{2 \overline{2}}+c_{4} \alpha^{2 \overline{3}}
\end{aligned}
$$

where $a_{i}, b_{j}, c_{k} \in \mathbb{C}$. We therefore need to establish the vanishing of nine coefficients, namely $a_{*}$ and $b_{2}, b_{5}, c_{1}, c_{4}$. (The new names $C, D,-A,-B$ for $b_{3}, b_{4}, c_{2}, c_{3}$ are carefully chosen to simplify formulae in subsequent sections.) We follow a type of decision tree in order to eliminate the coefficients one by one, but suppress the detailed calculations. The latter can be carried out by hand, though the procedure itself was refined by computer (Maple and Mathematica versions are available from the authors).

The system defined by (12) does not in general define a Lie algebra as $d^{2}$ (it is clearer to type this as $d d$ ) may not vanish. A valid solution must therefore satisfy both $d d \alpha^{i}=0$ and the SKT condition $\square \Omega=0$. On a computer, the operator $\square=-\frac{1}{2} d \circ(-i J) \circ d$ can be executed by treating $-i J$ as the substitution $\alpha^{\bar{j}} \mapsto-\alpha^{\bar{j}}$. If $d d=0$ then $\square=\partial \bar{\partial}$, but in general $\square \Omega$ may have a non-zero (3,1) component. To avoid this embarrassment, we shall restrict attention to

$$
\alpha^{i \bar{j}} \square \Omega=\alpha^{i} \wedge \alpha^{\bar{j}} \wedge \square \Omega
$$

which we identify with its coefficient relative to the standard 6 -form $\alpha^{123 \overline{123}}$. (Actually we shall only ever take $i=j$ in (13).)

A first calculation reveals that $\alpha^{1 \overline{1}} \square \Omega=x_{33}\left|c_{1}\right|^{2}$. The positive definiteness of $\Omega$ implies that $c_{1}=0$. Independently, the $\alpha^{23 \overline{2}}$ component of $d d \alpha^{3}$ equals $\left|c_{4}\right|^{2}$, so $c_{4}=0$. To simplify matters, we now consider two cases: $a_{5} \neq 0$ and $a_{5}=0$ (the choice of subscript 5 is partly a matter of taste).

Case 1. Suppose $a_{5} \neq 0$. Subtracting a multiple of $\alpha^{2}$ from $\alpha^{3}$ we may suppose that $b_{5}=0$. Using $d d \alpha^{3}$ (we mean of course 'the vanishing of $d d \alpha^{3}$ ') gives $c_{3}=0$. Using $d d \alpha^{2}$ now gives $a_{4}=0$ and $b_{1}=b_{2}=0$. To sum up, $a_{4}, b_{1}, b_{2}, b_{5}, c_{1}, c_{3}, c_{4}$ are all zero. If $p$ denotes the $\alpha^{13 \overline{1}}$ component of $d d \alpha^{3}$ then $p=a_{2} c_{2}+b_{4} \bar{a}_{5}$ and

$$
0=\alpha^{2 \overline{2}} \square \Omega=\left(\left|a_{2}\right|^{2}+\left|a_{5}\right|^{2}\right) x_{2 \overline{2}}-(\operatorname{Re} p) x_{3 \overline{3}} .
$$

This contradicts $a_{5} \neq 0$.

Case 2. Suppose $a_{5}=0$. We divide into two subcases: $c_{3} \neq 0$ and $c_{3}=0$.

Case 2.1. Suppose $c_{3} \neq 0$. Inspecting only $d d \alpha^{3}$ already gives $a_{1}=a_{2}=0$ and $b_{5}=0$. Using $d d \alpha^{2}$ then gives $a_{4}=0$. Returning to $d d \alpha^{3}$ gives $b_{2}=a_{3}=0$. To sum up, $a_{*}, b_{2}, b_{5}, c_{1}, c_{4}$ are all zero so we recover (2). The proof of Lemma 1.3 amounts to nothing more than an application of the equation $\alpha^{3 \overline{3}} \square \Omega=0$, which yields (3). In this way we arrive at the conclusion of Theorem 1.2 that represents the generic solution to the SKT hypothesis. 
Case 2.2. Suppose $c_{3}=0$. Using $d d \alpha^{2}$ shows that $a_{4}=0$ (even if $a_{1}=a_{2}=0$ ). We now divide into two subsubcases: $c_{2} \neq 0$ and $c_{2}=0$.

Case 2.2.1. Suppose $c_{2} \neq 0$. Using $d d \alpha^{2}$ and $d d \alpha^{3}$ respectively gives $a_{2}=0$ and $b_{5}=0$. Then $\alpha^{2 \overline{2}} \square \Omega=x_{3 \overline{3}}\left|b_{2}\right|^{2}$, whence $b_{2}=0$. Using $d d \alpha^{3}$ gives $a_{1}=0$. It follows that

$$
0=\alpha^{3 \overline{3}} \square \Omega=x_{3 \overline{3}}\left(\left|b_{1}\right|^{2}+\left|b_{4}\right|^{2}+\left|c_{2}\right|^{2}\right)
$$

and the solution reduces to

$$
\left\{\begin{array}{l}
d \alpha^{1}=0 \\
d \alpha^{2}=a_{3} \alpha^{1 \overline{1}} \\
d \alpha^{3}=b_{3} \alpha^{1 \overline{1}} .
\end{array}\right.
$$

By subtracting a multiple of $\alpha^{3}$ from $\alpha^{2}$ (or swapping the two if $b_{3}=0$ ), this solution can be subsumed into that of Theorem 1.2.

Case 2.2.2. Suppose $c_{2}=0$ so that $c_{*}=0$. The vanishing of $d d \alpha^{3}$ implies that $b_{5}=0$ and consequently that either $a_{1}=a_{2}=0$ or $b_{4}=0$. In the former case, the vanishing of $\alpha^{2 \overline{2}} \square \Omega$ and $\alpha^{3 \overline{3}} \square \Omega$ gives $b_{2}=0$ and $b_{1}=b_{4}=0$ respectively, and we obtain (14). The final situation to deal with is therefore that $a_{4}, a_{5}, b_{4}, b_{5}, c_{*}$ all vanish. This implies that

$$
\begin{aligned}
0=\alpha^{2 \overline{2}} \square \Omega & =x_{2 \overline{2}}\left|a_{2}\right|^{2}+x_{2 \overline{3}} a_{2} \bar{b}_{2}+x_{3 \overline{2}} \bar{a}_{2} b_{2}+x_{3 \overline{3}}\left|b_{2}\right|^{2} \\
& =i \Omega\left(a_{2} V_{2}+b_{2} V_{3}, \bar{a}_{2} \bar{V}_{2}+\bar{b}_{2} \bar{V}_{3}\right),
\end{aligned}
$$

in the notation of (5). Unless $a_{2}=b_{2}=0$, the restriction of $\Omega$ to the complex line spanned by $a_{2} V_{2}+b_{2} V_{3}$ is zero, which is impossible. Exactly the same argument applied to $\alpha^{33} \square \Omega$ gives $a_{1}=b_{1}=0$ and we are left with only $a_{3}, b_{3}$ non-zero, whence (14).

Remark 2.2. An invariant complex structure $J$ always induces a complex Lie algebra structure on the $i$-eigenspace $\mathfrak{g}^{1,0}$ of $\mathfrak{g}_{c}$. In the 6 -dimensional nilpotent case, $\mathfrak{g}^{1,0}$ is either abelian or isomorphic to the complex Heisenberg algebra. In the former case, $J$ is itself called abelian, and this is equivalent to asserting that $d$ maps the subspace $\Lambda^{1,0}$ of $\mathfrak{g}_{c}^{*}$ into $\Lambda^{1,1}$. The complex structure given by Theorem 1.2 is therefore abelian if and only if $E=0$. So SKT does not imply that the complex structure is abelian. This is in contrast with the result that if a 2-step nilpotent Lie group admits an invariant HKT structure then the hypercomplex structure must be abelian [8].

\section{Real Lie algebras}

Theorem 1.2 can be used to classify explicitly the real 6 -dimensional nilpotent Lie algebras admitting an SKT structure. Before explaining this, we shall introduce a formalism that will eventually help to understand and manipulate (2). Namely, we 
shall use complex $2 \times 2$ matrices to describe certain forms of type $(1,1)$ by setting

$$
\mathbf{Y}_{\alpha}=A \alpha^{\overline{1} 2}+B \alpha^{\overline{2} 2}+C \alpha^{1 \overline{1}}+D \alpha^{1 \overline{2}}
$$

where

$$
\mathbf{Y}=\left(\begin{array}{ll}
A & B \\
C & D
\end{array}\right)
$$

so that

$$
d \alpha^{3}=\mathbf{Y}_{\alpha}+E \alpha^{12}
$$

The exact positioning of the coefficients may seem strange, but follows a logic that is revealed in (25) below. The subscript ${ }_{\alpha}$ indicates the basis relative to which the construction is made.

Operations on 2-forms now translate into matrix operations in a natural way. For example,

$$
\mathbf{X}_{\alpha} \wedge \mathbf{Y}_{\alpha}=\operatorname{tr}\left(\mathbf{X} \mathbf{Y}^{\#}\right) \alpha^{1 \overline{1} 2 \overline{2}}
$$

where

$$
\mathbf{Y}^{\#}=\operatorname{adj} \mathbf{Y}=\left(\begin{array}{cc}
D & -B \\
-C & A
\end{array}\right)=(\operatorname{det} \mathbf{Y}) \mathbf{Y}^{-1}
$$

is the transpose of the matrix of cofactors. Moreover,

$$
\overline{\mathbf{Y}_{\alpha}}=\overline{\mathbf{Y}}_{\alpha}^{\#}
$$

and so

$$
\mathbf{Y}_{\alpha} \wedge \overline{\mathbf{Y}_{\alpha}}=\operatorname{tr}(\mathbf{Y} \overline{\mathbf{Y}}) \alpha^{1 \overline{1} 2 \overline{2}}
$$

Example 3.1. The complex coefficients $A, B, C, D$ are meant to be thought of as those in (2). In an illustration, we compute

$$
\begin{aligned}
d \alpha^{3} \wedge d \alpha^{\overline{3}} & =\left(\operatorname{tr}(\mathbf{Y} \overline{\mathbf{Y}})-|E|^{2}\right) \alpha^{1 \overline{1} 2 \overline{2}} \\
J d \alpha^{3} \wedge d \alpha^{\overline{3}} & =\left(\operatorname{tr}(\mathbf{Y} \overline{\mathbf{Y}})+|E|^{2}\right) \alpha^{1 \overline{1} 2 \overline{2}} \\
d \alpha^{3} \wedge d \alpha^{3} & =\operatorname{tr}(\mathbf{Y} \mathbf{Y} \#) \alpha^{1 \overline{1} 2 \overline{2}}=2(\operatorname{det} \mathbf{Y}) \alpha^{1 \overline{1} 2 \overline{2}}
\end{aligned}
$$

The middle equation is a more succinct version of the SKT formula (6).

The following result relies on the classification of [27], whose notation we freely adopt.

Theorem 3.2. A 6-dimensional nilmanifold $M=\Gamma \backslash G$ admits an invariant $S K T$ structure if and only if the Lie algebra $\mathfrak{g}$ is isomorphic to one of

$$
\begin{aligned}
& (0,0,0,0,13+42,14+23) \\
& (0,0,0,0,12,14+23) \\
& (0,0,0,0,12,34) \\
& (0,0,0,0,0,12) .
\end{aligned}
$$


In particular, $\mathfrak{g}$ is 2-step and $b_{1}(M) \geqslant 4$.

Proof. The possibility that $\mathfrak{g}$ is abelian is precluded by Definition 1.1. Vanishing of the real and imaginary components of $d \alpha^{1}, d \alpha^{2}$ in (2) implies immediately that $b_{1}(\mathfrak{g}) \geqslant 4$. Nomizu's theorem [25] tells us that the de Rham cohomology of the compact quotient $M$ is captured by that of the subcomplex of invariant forms, so $b_{1}(M)=b_{1}(\mathfrak{g})$. The fact that $d \alpha^{3} \in \bigwedge^{2}(\operatorname{ker} d)$ means (in the notation of [27]) that $\left(\mathfrak{g}^{2}\right)^{\mathrm{o}}=V_{2}$ equals $\mathfrak{g}$, which is therefore 2-step.

Using the methods of [27], any 2-step nilpotent Lie algebra with $b_{1} \geqslant 4$ is isomorphic to one of

$$
\begin{array}{lll}
\text { (i) }(0,0,0,0,12,13), & \text { (ii) } & (0,0,0,0,13+42,14+23), \\
\text { (iii) }(0,0,0,0,12,14+23), & \text { (iv) } & (0,0,0,0,12,34), \\
\text { (v) }(0,0,0,0,0,12), & \text { (vi) } & (0,0,0,0,0,12+34) .
\end{array}
$$

For example, in case (i) there is a real basis $\left(e^{i}\right)$ of 1 -forms for which $d e^{i}=0$ for $1 \leqslant i \leqslant 4, d e^{5}=e^{12}$ and $d e^{6}=e^{13}$. We need to eliminate (i) and (vi), and prove existence in the other cases.

Given (2), write $d \alpha^{3}=\sigma^{1}+i \sigma^{2}$ in real and imaginary components, and consider the real $2 \times 2$ matrix $\mathbf{B}=\left(b^{i j}\right)$ associated to the bilinear form

$$
\sigma^{i} \wedge \sigma^{j}=b^{i j} \alpha^{1 \overline{1} 2 \overline{2}}
$$

Under the SKT assumption, equations (19) give

$$
-\mathbf{B}=\left(\begin{array}{cc}
|E|^{2}+\operatorname{Re} U & \operatorname{Im} U \\
\operatorname{Im} U & |E|^{2}-\operatorname{Re} U
\end{array}\right)
$$

where $U=-\operatorname{det} \mathbf{Y}=B C-A D$. Using $d e^{5}, d e^{6}$ in place of $\sigma^{1}, \sigma^{2}$ is merely a change of real basis and must yield a matrix congruent to $\mathbf{B}$. It follows that, in the above examples,

$\mathbf{B}$ is the zero matrix for (i) and (v),

$\mathbf{B}$ has rank 1 for (iii) and (vi),

$\operatorname{det} \mathbf{B} \neq 0$ for (ii) and (iv).

In case (vi), we may rescale $\alpha^{3}$ so that $d \alpha^{3}$ is real. This implies that $d \alpha^{3}$ is a (1,1)-form and $E=0$. Since $\mathbf{B}$ has rank 1, the matrix (21) has zero determinant so $U=0$. This means that $\mathbf{B}=0$, which is a contradiction. In case (i) we already know that $\mathbf{B}=0$ so that $E=0=U$. Thus, $d \alpha^{3}=\mathbf{Y}_{\alpha}$ is a (1,1)-form with $\operatorname{rank} \mathbf{Y} \leqslant 1$. But the image $\left\langle e^{12}, e^{13}\right\rangle$ of $d$ in $\bigwedge^{2} g^{*}$ is divisible by the real 1 -form $e^{1}$ and must therefore be generated by $e^{1} \wedge J e^{1}$. But this contradicts the fact that $d\left(\mathfrak{g}^{*}\right)$ is actually 2 -dimensional.

The remaining cases are distinguished by the rank and signature of $\mathbf{B}$, and it is easy to check that the coefficients in (3) can be chosen to realize the four possibilities. 
Example 3.3. The irrelevance of the choice of Hermitian metric is special to the nilpotent situation. The third Lie algebra listed in Theorem 3.2 corresponds to the product $H_{3} \times H_{3}$ where $H_{3}$ is the real Heisenberg group. A simple example in which the SKT condition is metric dependent is provided by $H_{3} \times S^{3}$, where $S^{3}$ is identified with $S U(2)$. We may choose a real basis of 1-forms such that

$$
d e^{1}=0, \quad d e^{2}=0, \quad d e^{3}=e^{12}, \quad d e^{4}=e^{56}, \quad d e^{5}=e^{64}, \quad d e^{6}=e^{45} .
$$

Setting $\omega^{1}=e^{1}+i e^{2}, \omega^{2}=e^{3}+i e^{4}, \omega^{1}=e^{5}+i e^{6}$ gives

$$
\left\{\begin{array}{l}
d \omega^{1}=0 \\
d \omega^{2}=\frac{1}{2}\left(i \omega^{1 \overline{1}}-\omega^{3 \overline{3}}\right) \\
d \omega^{3}=\frac{1}{2}\left(\omega^{23}+\omega^{3 \overline{2}}\right) .
\end{array}\right.
$$

It follows that $d\left(\omega^{3 \overline{3}}\right)=0$ and $\partial \bar{\partial} \omega^{2 \overline{2}}=d \omega^{2} \wedge \overline{d \omega^{2}}=0$, so (11) satisfes $\square \Omega_{0}=0$. On the other hand, $\square \omega^{1 \overline{3}}=-\frac{1}{4} i \omega^{12 \overline{23}}$ and the general 2-form (4) determines an SKT metric if and only if $x_{1 \overline{3}}=-\overline{x_{3 \overline{1}}}=0$.

\section{Invariant forms on Iwasawa}

The real Lie algebra $(0,0,0,0,13+42,14+23)$ underlies the complex Heisenberg group $G$ and the Iwasawa manifold $M$, defined in the next paragraph. We shall summarize the relevant facts concerning invariant complex structures in this case, though important background for $\S \S 4,5$ can be found in $[21,27]$. The reader is implicitly referred to these papers for further explanation of a number of points.

Given

$$
G=\left\{\left(\begin{array}{ccc}
1 & z^{1} & z^{3} \\
0 & 1 & z^{2} \\
0 & 0 & 1
\end{array}\right): z^{i} \in \mathbb{C}\right\},
$$

we let $\Gamma$ be the discrete subgroup for which $z^{i}$ are Gaussian integers. Then $M$ is the set $\Gamma \backslash G=\{\Gamma g: g \in G\}$ of right cosets. It is a homogeneous space relative to the action of $G$ by right translation that persists on the quotient, though we shall be interested in the projections of tensors that are invariant by left translation on $G$.

The complex 1 -forms $\omega^{1}=d z^{1}, \omega^{2}=d z^{3}, \omega^{3}=-d z^{3}+z^{1} d z^{2}$ satisfy $d \omega^{3}=\omega^{12}$ and span the $(1,0)$ space $\Lambda_{0}$ of the bi-invariant complex structure $J_{0}$ on $M$. It is known that, in addition to $J_{0}$, any left-invariant complex structure on $G$ leaves invariant the real 4-dimensional subspace

$$
\mathbb{D}=\left\langle\operatorname{Re} \omega^{1}, \operatorname{Im} \omega^{1}, \operatorname{Re} \omega^{2}, \operatorname{Im} \omega^{2}\right\rangle
$$

that arises from a principal $T^{2}$-fibration $M \rightarrow T^{4}$. As a consequence, the generic invariant complex structure on $M$ has a space $\Lambda$ of $(1,0)$-forms generated by a 
basis of the type

$$
\left\{\begin{array}{l}
\alpha^{1}=\omega^{1}+a \omega^{\overline{1}}+b \omega^{\overline{2}} \\
\alpha^{2}=\omega^{2}+c \omega^{\overline{1}}+d \omega^{\overline{2}} \\
\alpha^{3}=\omega^{3}+x \omega^{\overline{1}}+y \omega^{\overline{2}}+u \omega^{\overline{3}}
\end{array}\right.
$$

with $a, b, c, d, x, y, u \in \mathbb{C}$. In any case, if $\Lambda=\left\langle\alpha^{1}, \alpha^{2}, \alpha^{3}\right\rangle$ is maximally complex it defines an invariant almost complex structure on $M$ that we denote by $J_{\mathbf{X}, x, y}$.

The effect of $J_{\mathbf{X}, x, y}$ on a real basis $\left(e^{i}\right)$ can be deduced by setting

$$
\omega^{1}=e^{1}+i e^{2}, \quad \omega^{2}=e^{3}+i e^{4}, \quad \omega^{3}=e^{5}+i e^{6},
$$

though little is to be gained from this, apart from verifying the real equations $d e^{5}=e^{13}+e^{42}$ and $d e^{5}=e^{14}+e^{23}$. The integrability condition for $J_{\mathbf{X}, x, y}$ is readily expressed in terms of (23) as $d \alpha^{3} \wedge \alpha^{123}=0$ (equivalently $d \alpha^{3} \wedge \alpha^{12}=0$ ). This reduces to

$$
u=b c-a d=-\operatorname{det} \mathbf{X}
$$

where

$$
\mathbf{X}=\left(\begin{array}{ll}
a & b \\
c & d
\end{array}\right)
$$

Using (16), we may consider the simple 2-form

$$
\begin{aligned}
\alpha^{12} & =\omega^{12}+a \omega^{\overline{1} 2}+b \omega^{\overline{2} 2}+c \omega^{1 \overline{1}}+d \omega^{1 \overline{2}}-u \omega^{\overline{12}} \\
& =\omega^{12}+c \omega^{1 \overline{1}}+d \omega^{1 \overline{2}}-a \omega^{2 \overline{1}}-b \omega^{2 \overline{2}}-u \omega^{\overline{12}} \\
& =\mathbf{X}_{\omega}+\omega^{12}-u \omega^{\overline{12}} .
\end{aligned}
$$

We write the characteristic polynomial of $\mathbf{X} \overline{\mathbf{X}}$ as $c(x)=x^{2}-\gamma x+\delta$, so that

$$
\gamma=\operatorname{tr}(\mathbf{X} \overline{\mathbf{X}}), \quad \delta=\operatorname{det}(\mathbf{X} \overline{\mathbf{X}})=|u|^{2}
$$

(notation of [21]).

The formulae

$$
\begin{aligned}
& \alpha^{1 \overline{1} 2 \overline{2}}=-\alpha^{12} \wedge \alpha^{\overline{12}}=(1-\gamma+\delta) \omega^{1 \overline{1} 2 \overline{2}}=c(1) \omega^{1 \overline{1} 2 \overline{2}} \\
& \alpha^{1 \overline{1} 2 \overline{2} 3 \overline{3}}=\alpha^{1 \overline{1} 2 \overline{2}} \wedge \alpha^{3 \overline{3}}=c(1)(1-\delta) \omega^{1 \overline{1} 2 \overline{2} 3 \overline{3}}
\end{aligned}
$$

express volume changes associated to a switch of basis from $\omega$ to $\alpha$. As a consequence, $\Lambda \cap \bar{\Lambda}=\{0\}$ if and only if

$$
\delta \neq 1 \quad \text { and } \quad c(1) \neq 0
$$

and these are the conditions that ensure that $J_{\mathbf{X}, x, y}$ is well defined. From now on, we assume that (24) and (28) hold. For simplicity, we also suppose $x=y=0$, and denote $J_{\mathbf{X}, 0,0}$ simply by $J_{\mathbf{X}}$. It will become obvious that reducing to this case causes no loss of generality, the key point being that $d \alpha^{3}$ (and so (31) below) does not involve $x, y$. An underlying reason for the irrelevance of $x, y$ is provided in $\S 5$.

One may invert (25) so as to express $\omega^{12}$ in terms of the $\alpha$ 's. Up to the overall factor $c(1)$, the corresponding formula is given by reversing the signs of $a, b, c, d$ : 
Lemma 4.1. $c(1) \omega^{12}=-\mathbf{X}_{\alpha}+\alpha^{12}-u \alpha^{\overline{12}}$.

Proof. Consider the bases $\left(\omega^{1}, \omega^{2}, \omega^{\overline{1}}, \omega^{\overline{2}}\right),\left(\alpha^{1}, \alpha^{2}, \alpha^{\overline{1}}, \alpha^{\overline{2}}\right)$. The second is related to the first by the block matrix

$$
\mathbf{M}=\left(\begin{array}{c|c}
\mathbf{I} & \mathbf{X} \\
\hline \overline{\mathbf{X}} & \mathbf{I}
\end{array}\right)
$$

in a row-by-row fashion. Set $\mathbf{Z}=(\mathbf{I}-\mathbf{X} \overline{\mathbf{X}})^{-1}$ so that $c(1)=\operatorname{det}\left(\mathbf{Z}^{-1}\right)$. By first observing that

$$
\mathbf{X} \overline{\mathbf{Z}}=\mathbf{Z}\left(\mathbf{Z}^{-1} \mathbf{X}\right) \overline{\mathbf{Z}}=\mathbf{Z}(\mathbf{I}-\mathbf{X} \overline{\mathbf{X}}) \mathbf{X} \overline{\mathbf{Z}}=\mathbf{Z X}(\mathbf{I}-\overline{\mathbf{X}} \mathbf{X}) \overline{\mathbf{Z}}=\mathbf{Z X}
$$

it is easy to verify that

$$
\mathbf{M}^{-1}=\left(\begin{array}{c|c}
\mathbf{Z} & -\mathbf{Z X} \\
\hline-\overline{\mathbf{X}} \mathbf{Z} & \overline{\mathbf{Z}}
\end{array}\right) .
$$

The coefficients $1, c, d,-a,-b,-u$ featuring in $(25)$ are the $2 \times 2$ minors of the $2 \times$ 4 matrix $\mathbf{M}=(\mathbf{I} \mid \mathbf{X})$, corresponding to the Mathematica command Minors[M, 2]. The coefficients of $\omega^{12}$ are therefore given by

$$
\text { Minors }[(\mathbf{Z} \mid-\mathbf{Z X}), 2]=(\operatorname{det} \mathbf{Z}) \operatorname{Minors}[(\mathbf{I} \mid-\mathbf{X}), 2] \text {, }
$$

using a well-known property of minors.

Proposition 4.2. The complex structure $J_{\mathbf{X}}$ is $S K T$ if and only if the eigenvalues of $\mathbf{X} \overline{\mathbf{X}}$ satisfy the equation

$$
\left(1+|z|^{2}\right)|1+z|^{2}=8|z|^{2}
$$

illustrated in the Figure.

It follows easily that all points of the curve are realized except for $z=1$ that is excluded by (28) (see Theorem 5.2 below).

Proof. We first prove that $J_{\mathbf{X}}$ is SKT if and only if

$$
1-6 \delta+\delta^{2}+\gamma+\gamma \delta=0
$$

Using Lemma 4.1,

$$
\begin{aligned}
c(1) d \alpha^{3} & =c(1) \omega^{12}+u c(1) \omega^{\overline{12}} \\
& =-\mathbf{X}_{\alpha}+\alpha^{12}-u \alpha^{\overline{12}}+u\left(-\overline{\mathbf{X}}_{\alpha}^{\#}+\alpha^{\overline{12}}-\bar{u} \alpha^{12}\right) \\
& =-\left(\mathbf{X}_{\alpha}+u \overline{\mathbf{X}}^{\#}{ }_{\alpha}\right)+(1-\delta) \alpha^{12} \\
& =\left(-\mathbf{X}+\delta \overline{\mathbf{X}}^{-1}\right)_{\alpha}+(1-\delta) \alpha^{12} .
\end{aligned}
$$

Comparing this with (18) yields

$$
c(1) \mathbf{Y}=-\mathbf{X}+\delta \overline{\mathbf{X}}^{-1}, \quad c(1) E=1-\delta .
$$


Thus

$$
c(1)^{2} \operatorname{tr}(\mathbf{Y} \overline{\mathbf{Y}})=\operatorname{tr}\left(\mathbf{X} \overline{\mathbf{X}}-2 \delta \mathbf{I}+\delta^{2}(\mathbf{X} \overline{\mathbf{X}})^{-1}\right)=\gamma-4 \delta+\delta^{2}(\gamma / \delta)
$$

and (30) follows from Lemma 1.3 and (19).

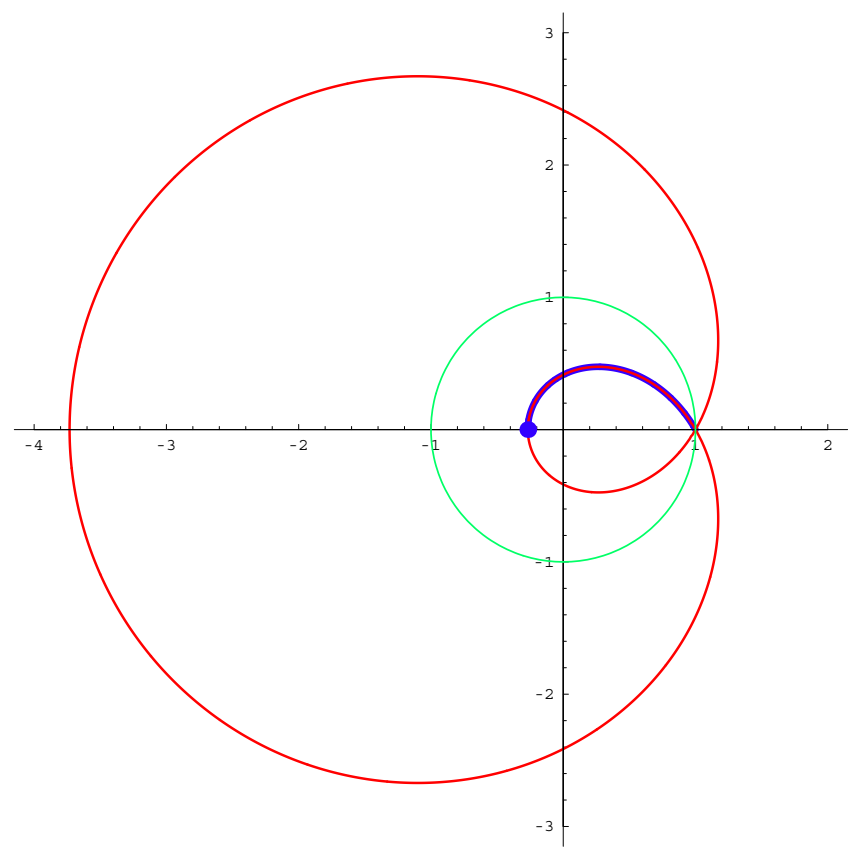

Figure. The curve (29) and the circle $|z|=1$.

Now let $\lambda, \mu$ denote the eigenvalues of $\mathbf{X} \overline{\mathbf{X}}$, so that $\gamma=\lambda+\mu$ and $\delta=\lambda \mu$. Thus, $\lambda \mu \geqslant 0$ so $\lambda$ and $\mu$ cannot be real with opposite signs. It is an elementary but non-trivial fact that if $\lambda, \mu$ are real and non-positive then they are equal $[18$, Problem 6, $\S 4.6]$. Under the SKT assumption,

$$
\gamma=\frac{-1+6 \delta-\delta^{2}}{1+\delta}
$$

is non-negative if and only if $3-2 \sqrt{2} \leqslant \delta \leqslant 3+2 \sqrt{2}$. The eigenvalues $\lambda, \mu$ can only be real if

$$
0 \leqslant(\lambda-\mu)^{2}=\gamma^{2}-4 \delta=\left(\frac{1-\delta}{1+\delta}\right)^{2}\left(\delta^{2}-14 \delta+1\right)
$$

which implies that $\delta \leqslant 7-\sqrt{48}$ or $\delta \geqslant 7+\sqrt{48}$. The various inequalities are incompatible, and there are no solutions with $\lambda, \mu>0$ and it follows that $\lambda=\bar{\mu}$. Thus,

$$
1-\gamma+\delta=1+\lambda+\bar{\lambda}+|\lambda|^{2}=|1+\lambda|^{2}>0
$$


and (30) translates into (29).

Remark 4.3. It is easy to check that equation (29) is unchanged by the substitution

$$
z \mapsto 1 / \bar{z}
$$

We shall see below that this corresponds to reversing the sign of $J$. Another curve with a similar shape invariant by both complex conjugation $z \mapsto \bar{z}$ and inversion $z \mapsto 1 / z$ is given in polar coordinates by $r=e^{\sin \theta}$. (This can be generalized by replacing $\sin \theta$ by an odd Fourier series.) If we shift the origin to the point $z=1$ of self-intersection, (29) becomes

$$
r=-3 \cos \theta \pm \sqrt{2+\cos ^{2} \theta}
$$

A true limaçon has the somewhat simpler equation $r=2 \cos \theta+1$, but the corresponding translate is not invariant under inversion.

\section{Moduli space interpretation}

Let $\mathcal{C}$ denote the set of all invariant complex structures on the Iwasawa manifold $M$. This is a subset of the set of all almost complex structures on $\mathfrak{g}$ (equivalently, maximally complex subspaces of $\mathfrak{g}_{c}$ ), that can in turn be identified with the homogeneous space $G L(6, \mathbb{R}) / G L(3, \mathbb{C})$. It is known that $\mathcal{C}$ has four connected components, and these can be described as follows.

Changing the sign of $J$ corresponds to an overall reversal of orientation, and corresponds to the transformation

$$
\left(\alpha^{1}, \alpha^{2}, \alpha^{3}\right) \mapsto\left(\alpha^{\overline{1}}, \alpha^{\overline{2}}, \alpha^{\overline{3}}\right)
$$

This identifies the components of $\mathcal{C}$ in pairs. Let $\hat{J}$ denote the restriction of $J$ to the real subspace $(22)$ underlying $\left\langle\omega^{1}, \omega^{2}\right\rangle$. The remaining two components of $\mathcal{C}$ are distinguished by the orientation of $\hat{J}$ or equivalently, by $(27)$, the sign of $c(1)$. For example

$$
\mathcal{C}_{+}=\left\{J_{\mathbf{X}, x, y}: x, y \in \mathbb{C},|u|<1, c(1)>0\right\}
$$

is the connected component that contains $J_{0}$.

To fully understand the SKT constraint on complex structures on $M$, we need to describe various group actions on $\mathcal{C}$ and their effect on the matrix $\mathbf{X}$.

(i) Involution. Referring to (23), can say that the almost complex structure $\hat{J}$ is represented by the matrix $(\mathbf{I} \mid \mathbf{X})$. This is replaced by

$$
(\overline{\mathbf{X}} \mid \mathbf{I}) \equiv\left(\mathbf{I} \mid \overline{\mathbf{X}}^{-1}\right)
$$

under (33) when $J, \hat{J}$ are replaced by $-J,-\hat{J}$. The equivalence relation $\equiv$ indicates premultiplication on both halves by an invertible matrix, and reflects a re-adjustment

$$
\left(\alpha^{\overline{1}}, \alpha^{\overline{2}}\right) \mapsto\left(\tilde{\alpha}^{1}, \tilde{\alpha}^{2}\right)
$$


into the row echelon form (23) in which $\tilde{\alpha}^{i}$ has leading term $\omega^{i}$. Notice that $\operatorname{det}\left(\overline{\mathbf{X}}^{-1}\right)=-1 / \bar{u}$, consistent with setting $\tilde{\alpha}^{3}=\alpha^{\overline{3}} / \bar{u}=\omega^{3}+\omega^{\overline{3}} / \bar{u}$. It now follows that

$$
-\left(J_{\mathbf{X}}\right)=J_{\overline{\mathbf{X}}^{-1}}
$$

If the eigenvalues of $\mathbf{X}$ are $\lambda, \bar{\lambda}$ then those of $\overline{\mathbf{X}}^{-1}$ are $1 / \bar{\lambda}, 1 / \lambda$, and this justifies Remark 4.3 and helps to explain why the solution curve has a limaçon shape. The inner part $|z|<1$ corresponds to solutions in the same component as $J_{0}$ and the outer part $|z|>1$ to the component of $-J_{0}$. One is an inversion of the other. The thicker part with $|z|<1$ and $\operatorname{Im} z \geqslant 0$ fits (after rotation by $45^{\circ}$ ) into the semicircular region of the diagram in [21], and represents the SKT solutions most faithfully. (The rest of the complex plane above does not fit into the diagram that is a schematic pasting of the real and complex plane best contemplated in 3 dimensions.)

The integrability condition for a left-invariant almost complex structure on an arbitrary Lie algebra $\mathfrak{g}$ is invariant under the action of the automorphism group

$$
\mathcal{G}=\{f \in \text { End } \mathfrak{g}:[f(v), f(w)]=f[v, w] .\}
$$

In the Iwasawa case, $\mathcal{G}$ can be identified with the semidirect product $G L(2, \mathbb{C}) \ltimes \mathbb{C}^{2}$ consisting of complex $3 \times 3$ matrices

$$
\left(\begin{array}{c|c}
\mathbf{P} & \mathbf{q} \\
\hline 0 & \operatorname{det} \mathbf{P}
\end{array}\right), \quad \mathbf{P} \in G L(2, \mathbb{C}), \quad \mathbf{q} \in \mathbb{C}^{2}
$$

(see [27]). The action on $\mathcal{C}$ of $\mathbf{P}$ and $\mathbf{q}$ can be considered separately.

(ii) Right translation. The normal subgroup $\mathbb{C}^{2}$ of $\mathcal{G}$ can be identified with the group $\operatorname{Ad} G$ of inner automorphisms of $\mathfrak{g}$. Since $J \in \mathcal{C}$ is by definition leftinvariant, $\operatorname{Ad}(g) J$ equals the right translate of $J$ by $g^{-1} \in G$. Whilst $J_{0}$ is fixed by this action ( $G$ being a complex Lie group), all the other orbits have positive dimension. Moreover, if $u \neq 0$ then $J_{\mathbf{X}, x, y}$ lies in the same orbit as $J_{\mathbf{X}}$ for any $x, y \in \mathbb{C}$. This is explained in [21], but to avoid duplication we next insert an infinitesimal version of this fact.

Remark 5.1. The action of the diffeomorphism group on a complex structure $J$ is detected by the image of

$$
\bar{\partial}: \Gamma\left(T \otimes \Lambda^{0,0}\right) \rightarrow \Gamma\left(T \otimes \Lambda^{0,1}\right)
$$

where $T$ denotes the holomorphic tangent bundle of $(M, J)$. Restricting to invariant tensors, a $p$-form $f$ with values in $T$ can be regarded as a linear mapping $\Lambda^{1,0} \rightarrow \Lambda^{p, 0}$ and $\bar{\partial} f$ is calculated by means of the formula

$$
(\bar{\partial} f)\left(\alpha^{i}\right)=\bar{\partial}\left(f\left(\alpha^{i}\right)\right)-f\left(\bar{\partial} \alpha^{i}\right)=-f\left(\bar{\partial} \alpha^{i}\right) .
$$


Since $\bar{\partial} \alpha^{3}=\mathbf{Y}_{\alpha}$, the dimension of $\operatorname{Im} \bar{\partial}$ coincides with the rank of $\mathbf{Y}$ which equals 2 if and only if $u \neq 0$ (see (18) and (32)). This discussion is relevant to the jumping of Hodge numbers discussed in [23].

In the SKT context, it is no restriction to impose the condition $\operatorname{det} \mathbf{X} \neq 0$ since $z=0$ is not a solution of (29). In the light of the above remarks, we shall call the structures $J_{\mathbf{X}, x, y}$ for which $\operatorname{det} \mathbf{X} \neq 0$ the stable points of $\mathcal{C}$ and indicate their entirety by $\mathcal{C}^{\text {st }}$. The quotient $\mathcal{C}^{\text {st }} / \operatorname{Ad} G$ is then smooth and injects into the moduli space of all complex structures on $M$ modulo diffeomorphism. For our calculations, we may always assume that $x=y=0$ so that $J=J_{\mathbf{X}}$ is completely determined by $\hat{J}$ and the matrix $\mathbf{X}$.

(iii) Outer automorphisms. The quotient $\mathcal{G} / \operatorname{Ad} G$ can be identified with $G L(2, \mathbb{C})$, an element of which acts by a change of basis

$$
\left\{\begin{array}{l}
\omega^{1} \mapsto p_{1}^{1} \omega^{1}+p_{2}^{1} \omega^{2} \\
\omega^{2} \mapsto p_{1}^{2} \omega^{1}+p_{2}^{2} \omega^{2}
\end{array}\right.
$$

By analogy to (35), the matrix $(\mathbf{I} \mid \mathbf{X})$ representing $\hat{J}$ is transformed into

$$
(\mathbf{P} \mid \mathbf{X} \overline{\mathbf{P}}) \equiv\left(\mathbf{I} \mid \mathbf{P}^{-1} \mathbf{X} \overline{\mathbf{P}}\right)
$$

and therefore a left action on $\mathcal{C}$ is defined by

$$
\mathbf{P}^{-1} \cdot J=J_{\mathbf{P}^{-1} \mathbf{X} \overline{\mathbf{P}}}
$$

The presence of $\operatorname{det} \mathbf{P}$ in (36) ensures that the extension from $\mathbb{D}$ to $\mathfrak{g}$ is well defined.

The remaining action by $G L(2, \mathbb{C})$ is less geometrical and the resulting quotient $\mathcal{C}^{\text {st }} / \mathcal{G}$ has singularities, an example of which is given after Example 5.3. Now

$$
\mathbf{X} \mapsto \mathbf{P}^{-1} \mathbf{X} \overline{\mathbf{P}}, \quad \mathbf{P} \in G L(n, \mathbb{C})
$$

is an action that gives rise to the theory of consimilarity for $n \times n$ matrices $\mathbf{X}$. If $\llbracket \mathbf{X} \rrbracket$ denotes the consimilarity class of $\mathbf{X}$ (i.e. an orbit for the above action) and $[\mathbf{Y}]$ the similarity class of $\mathbf{Y}$, there is a well defined mapping

$$
\phi: \llbracket \mathbf{X} \rrbracket \mapsto[\mathbf{X} \overline{\mathbf{X}}] .
$$

This is not a bijection as $\mathbf{X} \overline{\mathbf{X}}$ can be zero without $\mathbf{X}$ being zero. However, the general theory of consimilarity developed in $[18, \S 4.6]$ implies that $\phi$ restricts to a bijection between classes subject to an equal rank condition. In the simple case of $n=2$, we may condense the discussion of this section into

Theorem 5.2. An invariant complex structure $J$ on the Iwasawa manifold is $S K T$ if and only if it equals $J_{\mathbf{X}, x, y}$ where

$$
\mathbf{X}=\mathbf{P}^{-1}\left(\begin{array}{ll}
0 & z \\
1 & 0
\end{array}\right) \overline{\mathbf{P}}
$$

with $\mathbf{P} \in G L(2, \mathbb{C}), z$ a solution of (29) different from 1 and $x, y \in \mathbb{C}$. Moreover, such structures lie in the connected components of $J_{0}$ and $-J_{0}$ in $\mathcal{C}$. 
Proof. If $\mathbf{X}$ has the form given then $\mathbf{X} \overline{\mathbf{X}}$ is similar to a diagonal matrix with entries $z, \bar{z}$. Since $z \neq 1, J_{\mathbf{X}, x, y}$ is well defined. Bearing in mind (from (ii)) that $x, y$ are irrelevant, Proposition 4.2 implies that $J_{\mathbf{X}, x, y}$ is SKT.

Conversely, suppose that $J \in \mathcal{C}$ is SKT. The complex structures $J_{\mathbf{X}, x, y}$ represented by (23) constitute a dense set of $\mathcal{C}$, and the only missing points are those arising when one or more of the coefficents become infinite. The SKT condition involves only $d \alpha^{3}$, so the only case potentially not covered by (23) is that in which $\alpha^{3}$ belongs to the span of $\omega^{\overline{3}}$ and $\mathbb{D}$. But then $J d \alpha^{3} \wedge d \alpha^{\overline{3}} \neq 0$ and $J$ cannot be SKT by Lemma 1.3. The statement about connected components now follows from the fact that the solutions in Proposition 4.2 all satisfy $c(1)>0$ (see (34)).

We may therefore suppose that $J=J_{\mathbf{X}, 0,0}=J_{\mathbf{X}}$, and that the eigenvalues $z, \bar{z}$ of $\mathbf{X} \overline{\mathbf{X}}$ satisfy (29). Assume firstly that $z$ is not real. Choose $\mathbf{P} \in G L(2, \mathbb{C})$ such that $\mathbf{Y}=\mathbf{P} \mathbf{X} \overline{\mathbf{P}}^{-1}$ satisfies

$$
\mathbf{Y} \overline{\mathbf{Y}}=\mathbf{P}(\mathbf{X} \overline{\mathbf{X}}) \mathbf{P}^{-1}=\left(\begin{array}{cc}
z & 0 \\
0 & \bar{z}
\end{array}\right) .
$$

In the notation (17), $A \bar{B}+B \bar{D}=0=A \bar{C}+C \bar{D}$, which implies

$$
A(B \bar{C}-\bar{B} C)=0=D(B \bar{C}-\bar{B} C)
$$

and $A=D=0$. We can now premultiply $\mathbf{P}$ by a diagonal matrix so as to convert $B$ to $z$ and $C$ to 1 , as required.

Now assume that $z \in \mathbb{R}$. The equation $\mathbf{Y} \overline{\mathbf{Y}}=\left(\begin{array}{ll}z & 0 \\ 1 & z\end{array}\right)$ would imply that $B=0$ and $z>0$, which contradicts (29). Thus, $\mathbf{X} \overline{\mathbf{X}}$ is again diagonalizable and we need to solve

$$
\mathbf{Y} \overline{\mathbf{Y}}=z \mathbf{I},
$$

that implies that $|A|=|D|$. But we can find $\mathbf{Q} \in G L(2, \mathbb{C})$ such that $\tilde{\mathbf{Y}}=\mathbf{Q} \mathbf{Y} \overline{\mathbf{Q}}^{-1}$ has its last entry $\tilde{D}$ zero. Since $\tilde{\mathbf{Y}}$ satisfies (39) in place of $\mathbf{Y}$, we also have $\tilde{A}=0$, and we can modify $\mathbf{Q}$ so that $\tilde{C}=1$ and $\tilde{\mathbf{Y}}=\left(\begin{array}{ll}0 & z \\ 1 & 0\end{array}\right)$.

Example 5.3. The point $z=1$ on the curve is not admissible, because in this case

$$
\alpha^{1}=\omega^{1}+\omega^{\overline{2}}=\overline{\alpha^{2}},
$$

and any corresponding tensor $J$ is degenerate. The solutions

$$
z=-2 \pm \sqrt{3} \in \mathbb{R}
$$

are admissible, but since $-2-\sqrt{3}=1 /(-2+\sqrt{3})$ the $J$ 's coincide up to an overall complex conjugation, in accordance with the discussion in (i). These are the simplest solutions to the SKT equation. The purely imaginary solutions are $z= \pm i \sqrt{3 \pm 2 \sqrt{2}}$, and we presume that there are no rational solutions to (29). 
Let $H_{z}$ denote the stabilizer of $\left(\begin{array}{ll}0 & z \\ 1 & 0\end{array}\right)$ in $G L(2, \mathbb{C})$ for the action (37). It is easy to verify that if $z \in \mathbb{R}$ and $z=-x<0$ then

$$
H_{z}=\left\{\left(\begin{array}{cc}
a & -\bar{c} x \\
c & \bar{a}
\end{array}\right):|a|^{2}+|b|^{2} \neq 0\right\} \cong G L(1, \mathbb{H})
$$

If $z \in \mathbb{C} \backslash \mathbb{R}$ then $H_{z}$ is isomorphic to the common subgroup $\mathbb{C}^{*}$ obtained by setting $c=0$ in (40). It follows that the SKT structures $z=-2 \pm \sqrt{3}$ (one of which is a blob in the Figure) represent singular points in $\mathcal{C}^{\text {st }} / \mathcal{G}$.

\section{Examples with reduced holonomy}

Let $M$ be a Hermitian manifold of dimension $2 n$, and $\eta$ an $(n, 0)$-form on $M$ of constant norm. Let $\nabla$ be an arbitrary Hermitian connection on $M$, so that $\nabla J=0$ and $\nabla g=0$, and denote its torsion tensor by $T$. Then

$$
\nabla \eta=\beta \otimes \eta,
$$

where $\beta \in \mathfrak{u}(1)$ is a purely imaginary 1-form. Anti-symmetrizing,

$$
\beta^{0,1} \wedge \eta=d \eta+(T \cdot \eta)^{n, 1},
$$

where - stands for a suitable linear mapping. The terms on the right-hand side of (41) can be identified with the respective intrinsic torsion components $W_{5}, W_{4}$ [5].

As an application of (41), we may deduce the following well-known result. The Chern connection is the unique Hermitian connection for which $T$ has no $(1,1)$ component, so that

$$
T(\cdot, \cdot)+T(J \cdot, J \cdot)=0 .
$$

This means that $T$ takes values in the real vector space underlying $\Lambda^{1,0} \oplus \operatorname{End}\left(\Lambda^{1,0}\right)$, and we have $(T \cdot \eta)^{n, 1}=0$. Thus, a closed (equivalently, holomorphic) $(n, 0)$-form of constant norm is necessarily parallel with respect to the Chern connection. For more details, see $[2,9,20]$.

For the remainder of this paper, let $\nabla$ denote the Bismut connection and $D$ the Levi-Civita connection. Recall that $\nabla$ is the unique connection for which $g$ and $J$ are parallel and the torsion $g(\cdot, T(\cdot, \cdot))$ is totally skew-symmetric. The following result is closely related to formula (2.7) of [2].

Proposition 6.1. Let $M$ be a compact nilmanifold with an invariant Hermitian metric. The holonomy of $\nabla$ reduces to $S U(n)$ if and only if $M$ is balanced.

Proof. The basis arising from Theorem 2.1 furnishes us with a closed $(n, 0)$-form

$$
\eta=\alpha^{12 \cdots n}
$$

of constant norm. To say that the holonomy of $\nabla$ is contained in $S U(n)$ means that there exists an $(n, 0)$ form $\xi$ satisfying $\nabla \xi=0$. This equation implies that $\xi$ 
also has constant norm, so $\eta=e^{i h} \xi$ for some function $h: M \rightarrow \mathbb{R} / 2 \pi \mathbb{Z}$, and (41) is valid with $\beta=i d h$.

Since $T$ is determined by $d \Omega$, the last term of (41) determines a non-zero $U(n)$ equivariant mapping

$$
\Lambda^{1,2} \otimes \Lambda^{n, 0} \rightarrow \Lambda^{n, 1} \cong \Lambda^{n-1,0} .
$$

This is equivalent to the $S U(n)$-equivariant contraction $\Lambda^{1,2} \rightarrow \Lambda^{0,1} \cong \Lambda^{n-1,0}$ that extracts the 'trace' of $\bar{\partial} \Omega$. A comparison with (8) (which corresponds to the case $\beta=2 d f$ ) reveals that $\theta$ is proportional to $J d h$. Using $(7)$, it follows that $d d^{*} \Omega=0$ and, by integration, $d^{*} \Omega=0$.

Conversely, if $\theta=0$ then (41) implies that (43) is itself parallel for $\nabla$, so the global holonomy group reduces to $S U(n)$.

Returning to six dimensions, it is an easy matter to list Lie algebras admitting a balanced Hermitian metric, so that $\nabla$ has reduced holonomy. We restrict attention to the algebras considered in $\S 3$. Case (v) is realized by taking $C=1$ and $A, B, D, E$ zero which is incompatible with (9). But this is the only one excluded:

Corollary 6.2. Each of the Lie algebras

$$
\begin{aligned}
& (0,0,0,0,13+42,14+23) \\
& (0,0,0,0,12,14+23) \\
& (0,0,0,0,12,34) \\
& (0,0,0,0,0,12,13) \\
& (0,0,0,0,0,12+34)
\end{aligned}
$$

admits a Hermitian structure for which the holonomy group of $\nabla$ is a subgroup of $S U(3)$.

Proof. Relative to the complex basis $\left(\alpha^{i}\right)$ considered in $\S 1$, the Hermitian structure will be the standard one corresponding to (11). The balanced condition $B=C$ is then independent of $A, D, E$, and this gives us the flexibility to realize the various cases as follows. If $B=C=0$ then

$A=D=0$ and $E=1$ gives case (ii),

$A=D=1$ and $E=0$ gives (vi),

$A=E=1$ and $D=0$ gives (i),

$A=D=1$ and $E=2$ gives (iii).

The real basis used to define each of the Lie algebras listed above may bear a complicated relation to $\left(\alpha^{i}\right)$, as in the remaining case that corresponds to the direct sum of two real Heisenberg algebras. Nonetheless, it is easy to check that if $B=C=i$ then

$A=1, D=-1$ and $E=2$ gives (iv)

up to an isomorphism of real Lie algebras. 
Example 6.3. The holonomy group will generally equal $S U(3)$ in the situation described by Corollary 6.2, even if one replaces $\nabla$ by another canonical Hermitian connection. But a further reduction takes place in case (vi), namely $(0,0,0,0,0,12+34)$, precisely for the Bismut connection. To explain this, let $\left(\alpha^{i}\right)$ be a basis of $(1,0)$-forms satisfying $d \alpha^{3}=\alpha^{\overline{1} 2}+\alpha^{1 \overline{2}}$ as in the last proof, and set

$$
\alpha^{1}=\frac{1}{2}\left(e^{1}+i e^{3}\right), \quad \alpha^{2}=\frac{1}{2}\left(e^{2}+i e^{4}\right), \quad \alpha^{3}=\frac{1}{2}\left(e^{6}+i e^{5}\right) .
$$

Relative to the Hermitian structure defined by (11), the Riemannian metric is locally reducible with $D e^{5}=0$, and we claim that

$$
\nabla \alpha^{1}=e^{6} \otimes \alpha^{2}, \quad \nabla \alpha^{2}=-e^{6} \otimes \alpha^{1}, \quad \nabla \alpha^{3}=0 .
$$

These equations define a connection for which $\nabla g=0$ and $\nabla J=0$; indeed the matrix of 1-forms relative to $\left(\alpha^{i}\right)$ has values in $\left\langle e^{6}\right\rangle \otimes \mathfrak{u}(1)$. Furthermore, the torsion $g(\cdot, T(\cdot, \cdot))$ equals

$\sum_{i=1}^{3} e^{i} \otimes\left(\wedge \nabla e^{i}-d e^{i}\right)=e^{1} \otimes e^{62}+e^{3} \otimes e^{64}-e^{2} \otimes e^{61}-e^{4} \otimes e^{63}-e^{6} \otimes e^{12}-e^{6} \otimes e^{34}$,

and is totally antisymmetric as claimed.

The reduction of the holonomy of $\nabla$ to $S U(n)$ is equivalent to the vanishing of the 2 -form

$$
\rho(X, Y)=\frac{1}{2} \sum_{i=1}^{n} g\left(R_{X Y} e_{i}, J e_{i}\right), \quad X, Y \in \mathfrak{g}
$$

where $R$ is the curvature tensor of $\nabla$. This is not in general equal to the Ricci tensor of $g$ when there is non-zero torsion, though the situation is just as intriguing for the Levi-Civita connection of pseudo-Riemannian metric $h$.

Lemma 6.4. Suppose that $(J, h)$ is an invariant pseudo-Kähler structure on a nilmanifold $M$ of real dimension $2 n$. Then the Ricci tensor of $h$ vanishes.

Proof. The hypothesis means that $M$ admits both an invariant complex structure $J$ and a closed 2-form $\Omega$ for which $h$ (defined by (1) with $h$ in place of $g$ ) is a pseudo-Riemannian metric. In the presence of a compatible complex structure, the equation $d \Omega=0$ is sufficient to imply that $D \Omega=0$ and thus $D J=0$, just as in the familiar (positive-definite) Kähler case. We can therefore apply an equation of type (41) to deduce that $D \eta=0$, whence the result.

As an example,

Proposition 6.5. The Lie algebra $(0,0,0,0,13+42,14+23)$ associated to the Iwasawa manifold admits a pseudo-Kähler metric $h$ which is Ricci-flat but not flat. 
Proof. We take

$$
\Omega=e^{16}+e^{25}+e^{34}, \quad J e^{1}=e^{2}, J e^{3}=-e^{4}, J e^{5}=-e^{6} .
$$

It is necessary to check that

$$
d \Omega=-e^{1} \wedge d e^{6}-e^{2} \wedge d e^{5}=-e^{1} \wedge\left(e^{14}+e^{23}\right)-e^{2} \wedge\left(e^{13}+e^{42}\right)=0
$$

and that

$$
J \cdot \Omega=\left(J e^{1}\right) \wedge\left(J e^{6}\right)+\left(J e^{2}\right) \wedge\left(J e^{5}\right)+\left(J e^{3}\right) \wedge\left(J e^{4}\right)=\Omega .
$$

Setting $\alpha^{1}=e^{1}+i e^{2}, \alpha^{2}=e^{3}-i e^{4}, \alpha^{3}=e^{5}-i e^{6}$ gives $d \alpha^{3}=\alpha^{1 \overline{2}}$, so $J$ is integrable and abelian. Observe that $\hat{J}$ has negative orientation, so its connected component in $\mathcal{C}$ does not contain $\pm J_{0}$. The resulting pseudo-metric (1) assumes the matrix form

$$
\left(h_{i j}\right)=\left(\begin{array}{cccccc}
0 & 0 & 0 & 0 & 1 & 0 \\
0 & 0 & 0 & 0 & 0 & -1 \\
0 & 0 & 1 & 0 & 0 & 0 \\
0 & 0 & 0 & 1 & 0 & 0 \\
1 & 0 & 0 & 0 & 0 & 0 \\
0 & -1 & 0 & 0 & 0 & 0
\end{array}\right)
$$

and has signature $(4,2)$.

A computation reveals that

$$
R_{1212}=\sum_{m=1}^{6} h_{1 m} R^{m}{ }_{212}=R_{212}^{5}=-2,
$$

and so the full Riemann curvature is non-zero.

A contrasting situation is provided by the Lie algebra of Example 6.3 with $b_{1}=5$. For $(0,0,0,0,0,12+34)$ carries no invariant symplectic form [27], and does not therefore possess an invariant pseudo-Kähler metric. We suspect that the remaining four algebras in (20) do admit pseudo-Kähler metrics.

A completely different class of Ricci-flat structures on nilmanifolds, indeed ones with signature $(2 n, 2 n)$ that are not invariant, are discussed in [10].

\section{References}

[1] E. Abbena, S. Garbiero and S. Salamon, Almost Hermitian geometry of 6-dimensional nilmanifolds, Ann. Sc. Norm. Sup. 30 (2001), 147-170.

[2] B. Alexandrov and S. Ivanov, Vanishing theorems on Hermitian manifolds, Differ. Geom. Appl. 14 (2001), 251-265.

[3] C. Benson and C. S. Gordon, Kähler and symplectic structures on nilmanifolds, Topology 27 (1988), 513-518.

[4] J.-M. Bismut, A local index theorem for non-Kähler manifolds, Math. Ann. 284 (1989), 681-699. 
[5] S. Chiossi and S. Salamon, The intrinsic torsion of $S U(3)$ and $G_{2}$ structures, in: Differential Geometry, Valencia 2001, 115-133, World Scientific, 2002.

[6] L. A. Cordero, M. Fernández, A. Gray and L. Ugarte, Compact nilmanifolds with nilpotent complex structures: Dolbeault cohomology, Trans. Amer. Math. Soc. 352 (2000), 54055433.

[7] P. Deligne, P. Griffiths, J. Morgan and D. Sullivan, Real homotopy theory of Kähler manifolds, Invent. Math. 29 (1975), 245-274.

[8] I. G. Dotti and A. Fino, HyperKähler torsion structures invariant by nilpotent Lie groups, Classical Quantum Gravity 19 (2002), 551-562.

[9] A. Fino and G. Grantcharov, On some properties of the manifolds with skew-symmetric torsion and holonomy $S U(n)$ and $S p(n)$, Advances in Math., to appear.

[10] A. Fino, H. Pedersen, Y. S. Poon and M. Sørensen, Neutral Calabi-Yau structures on Kodaira manifolds, Preprint, 2002, http://www.imada.sdu.dk

[11] S. J. Gates, Jr., C. M. Hull and M. Roček, Twisted multiplets and new supersymmetric nonlinear $\sigma$-models, Nuclear Phys. B 248 (1984), 157-186.

[12] P. Gauduchon, La 1-forme de torsione d'une variété hermitienne compacte, Math. Ann. 267 (1984), 495-518.

[13] P. Gauduchon, Hermitian connections and Dirac operators, Boll. Un. Mat. Ital. B 11 (1997), 257-288.

[14] G. Grantcharov, C. McLaughlin, H. Pedersen and Y. S. Poon, Lie groups, Kodaira manifolds and complex deformation, Glasgow Math. J., to appear.

[15] A. Gray and L. M. Hervella, The Sixteen classes of almost Hermitian manifolds and their linear invariants, Ann. Mat. Pura Appl. 123 (1980), 35-58.

[16] J. Gutowski, S. Ivanov and G. Papadopoulos, Deformations of generalized calibrations and compact non-Kähler manifolds with vanishing first Chern class, Asian J. Math. 7 (2003), 39-79.

[17] K. Hasegawa, Minimal models of nilmanifolds, Proc. Amer. Math. Soc. 106 (1989), 65-71.

[18] R. A. Horn and C. R. Johnson, Matrix Analysis, Cambridge University Press, 1985.

[19] P. S. Howe and G. Papadopoulos, Further remarks on the geometry of two-dimensional nonlinear $\sigma$ models, Classical Quantum Gravity 5 (1988), 1647-1661.

[20] S. Ivanov and G. Papadopoulos, Vanishing theorems and string backgrounds, Classical Quantum Gravity 18 (2001), 1089-1110.

[21] G. Ketsetzis and S. Salamon, Complex structures on the Iwasawa manifold, Adv. in Geom. 4 (2004), 165-179.

[22] A. I. Malcev, On a class of homogeneous spaces, Amer. Math. Soc. Translation Ser. 19 (1962), 276-307.

[23] I. Nakamura, Complex parallelizable manifolds and their small deformations, J. Differ. Geom. 10 (1975), 85-112.

[24] J. Neisendorfer and L. Taylor, Dolbeault homotopy theory, Trans. Amer. Math. Soc. 245 (1978), 183-210.

[25] K. Nomizu, On the cohomology of compact homogeneous spaces of nilpotent Lie groups, Ann. of Math. 59 (1954), 531-538.

[26] G. Papadopoulos, KT and HKT geometries in strings and in black hole moduli spaces, Preprint, 2002, hep-th/0201111.

[27] S. Salamon, Complex structures on nilpotent Lie algebras, J. Pure Appl. Algebra 157 (2001), 311-333.

[28] Ph. Spindel, A. Sevrin, W. Troost and A. Van Proeyen, Complex structures on parallelised group manifolds and supersymmetric $\sigma$-models, Phys. Lett. B 206 (1988), 71-74.

[29] A. Strominger, Superstrings with torsion, Nuclear Phys. B 274 (1986), 253-284.

[30] K. Yano, Differential geometry on complex and almost complex spaces, International Series of Monographs in Pure and Applied Mathematics 49, Macmillan, New York, 1965. 
A. Fino

Dipartimento di Matematica

Università di Torino

Via Carlo Alberto 10

10124 Torino

Italy

e-mail: annamaria.fino@unito.it

S. Salamon

Dipartimento di Matematica

Politecnico di Torino

Corso Duca degli Abruzzi 24

10129 Torino

Italy

e-mail: salamon@calvino.polito.it

(Received: October 14, 2002)
M. Parton

Dipartimento di Scienze

Università di Chieti-Pescara

Viale Pindaro 87

65127 Pescara

Italy

e-mail: parton@sci.unich.it

(12) To access this journal online:

(4) http://www.birkhauser.ch 